International Journal of Engineering \& Technology, $7(4.20)(2018) 251-258$
International Journal of Engineering \& Technology
SPC
Website www.sciencepubco.com/index.php/IJET
Research paper

\title{
Applying Various Types of Loading on Continuous Deep Beams Using Strut and Tie Modelling
}

\author{
Khattab Saleem Abdul-Razzaq $^{1 *}$, Ali Mustafa Jalil ${ }^{2}$, Abbas H. Mohammed ${ }^{3}$ \\ ${ }^{1}$ University of Diyala, Civil Engineering, Diyala, Iraq \\ ${ }^{2}$ University of Diyala, Civil Engineering, Diyala, Iraq \\ ${ }^{3}$ University of Diyala, Civil Engineering, Diyala, Iraq \\ *Corresponding author E-mail: dr.khattabsaleem@yahoo.com
}

\begin{abstract}
This work aims at presenting detailed procedures companied by numerical examples for designing reinforced concrete two span continuous deep beams under various types of loading; one concentrated force, two concentrated forces and uniform load for each span. Analysis and design was conducted based on Strut and Tie modeling (STM) of ACI 318M-14 since they contain significant extents of D-regions and they show a marked truss or tied arch action. It was found that changing the loading type has a significant impact on the capacity for the same specimen that has the same dimensions, concrete and steel properties, in addition to the same amount and arrangement of steel reinforcement. In more detail, the increase in the number of concentrated forces causes an obvious increase in ultimate capacity due to the reduction in span to overall height $(\mathrm{a} / \mathrm{h})$ ratio and the increase in the value of the strut-tie angle, which causes shortening in the length of the strut. Therefore, the ultimate capacity increased by about (44-70) \% when the applied load was changed from 1-concentrated force to 2-concentrated forces or to uniformly distributed load.
\end{abstract}

Keywords: Reinforced concrete, Continuous deep beams, Strut and Tie, one and two concentrated forces, Uniform load, Design procedures.

\section{Introduction}

Deep beams are loaded on one face and supported on the opposite face such that strut-like compression elements can develop between the loads and supports and that satisfies (a) or (b) [1]: (a) Clear span ln does not exceed four times the overall member depth $\mathrm{h}$. (b) Concentrated loads exist within a distance $2 \mathrm{~h}$ from the face of the support. Many investigators have suggested empirical and semiempirical expressions to determine the ultimate load capacity of conventionally reinforced concrete deep beams [2, 3]. Some researchers studied the parameters that affect deep beams such as effect of heating, existence of openings, strengthening of openings, amount and type of web reinforcement, types of loading, concrete and steel strengths [4-10]. Furthermore, Abdul-Razzaq and Jebur suggested alternatives for reinforced concrete deep beams by reinforcing struts and ties only as compressive and tensile members, respectively [11]. Since 2002, the ACI- 318 Code procedure is based on empirical equations for the design of deep beams. According to ACI 318M-14 [1], STM is defined as "a truss model of a structural member or of a D- region in such a member, made up of struts and ties connected at nodes, capable of transferring the factored loads to the supports or to adjacent B-regions". Provisions for STM have been also taken into considerations by many authors for the design purposes. STM complies with the plasticity lower bound theory, which needs that only yield conditions in addition to equilibrium to be satisfied. Plasticity lower bound theory states that if the load has such a value that it is possible to find a distribution of stress corresponding to stresses that keep internal and external equilibrium within the yield surface, then this load will not cause failure of the body. In other words, the capacity of a structure as estimated by a lower bound theory will be less than or equal to the real failure load of the body in question [12].

\section{STM Analysis and Design Procedure}

An emerging methodology for the design of all types of D-Regions is to predict and design an internal truss. This truss is consisting of steel tension ties and concrete compressive struts that are interconnected at nodes, to support the imposed loading through the regions of discontinuity. The STM design procedure includes the general steps summarized below [1]:

i. Define the D-Region boundaries and determine the imposed sectional and local forces.

ii. Draw the internal supporting truss, find equivalent loadings, and calculate the truss member forces.

iii. Choose the reinforcing steel to provide the necessary capacity of the tie and ensure that this tie reinforcement is adequately anchored in the nodal zones.

iv. Evaluate the dimensions of the nodes and struts, such that the capacities of these components are adequate to carry the values of the design forces.

v. Select the distributed reinforcement to guarantee the ductile behavior of the D-Regions. It is important to note that both hydrostatic and non-hydrostatic nodes are idealizations of reality. The use of either hydrostatic or non-hydrostatic nodes is an assumption that a design tool intended to provide a simple method for proportioning STM. The classical method of node dimensioning is by node shape arranging so that the applied stresses on all sides of the node are equal. The stress biaxial 
state in the node is hydrostatic; so, the in-plane stresses are homogeneous, isotropic, and equal to those on the sides. Arranging the node in this shape can be made by sizing the node boundaries so that they become proportional and perpendicular to the forces that acting on them [13].

Based on the above, three specimens were designed to study the behavior of reinforced concrete deep beams under various types of loading. In order to recognize designation of specimens easily, Table 1 shows the way followed in this designation.

Table 1: Designation way of specimens

\begin{tabular}{|c|c|}
\hline Letter & Meaning \\
\hline CD & Conventional $\underline{\text { Continuous Deep Beam }}$ \\
\hline 1F & Subjected to $\underline{\text { 1-Concentrated Force }}$ \\
\hline 2F & Subjected to $\underline{\mathbf{2}}$-Concentrated Forces \\
\hline UL & Subjected to $\underline{\text { Uniformly Distributed Load }}$ \\
\hline
\end{tabular}

\section{Three Loading Cases}

\subsection{One Concentrated Force}

Fig. 1 shows the principal stress paths and the assumed truss under 1-concentrated force in the continuous deep beam specimen CD.1F. The geometry should be conformed to the deep beam definition $l n \leq$ $4 h$ [1]. Moreover, the minimum web reinforcement ratios for both horizontal and vertical ones should be 0.0025 with the maximum spacing of $d / 5$, which is not more than $300 \mathrm{~mm}$ [1]. Finally, capacity was checked for each node face, the idealized bottle shape diagonal strut, in addition to top and bottom ties.

To analyze the deep beam with one concentrated force, the steps shown in Fig. 2 may be followed. Details for CD.1F specimen are illustrated in Fig.3

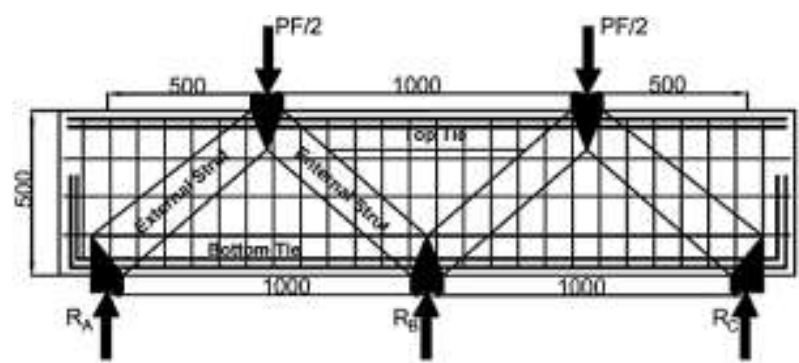

Fig. 1: The principal stress paths and the assumed truss for CD.1F, all dimensions in $\mathrm{mm}$

\subsubsection{Numerical Example No. 1, 1-Concentrated Force:}

Sample calculations for predicting failure load depending on strut and tie method for reinforced concrete continuous deep beam specimen that subjected to one central concentrated force CD.1F is presented here.

$\mathrm{h}=500 \mathrm{~mm}, l c=1000 \mathrm{~mm}, \mathrm{~d}=440.5 \mathrm{~mm}, a=500 \mathrm{~mm}, b_{w}=150 \mathrm{~mm}$, $\mathrm{f}^{\prime} \mathrm{c}=30 \mathrm{MPa}$, bearing plate dimensions $=(100 * 150) \mathrm{mm}$, main longitudinal top and bottom reinforcement $=4 \phi 16 \mathrm{~mm}, \mathrm{f}_{\mathrm{y}}=500$ $\mathrm{MPa}$, vertical web reinforcement $=\phi 6 \mathrm{~mm} @ 85 \mathrm{~mm} \mathrm{c} / \mathrm{c}, \mathrm{f}_{\mathrm{yv}}=440$ $\mathrm{MPa}$, horizontal web reinforcement $=\phi 6 \mathrm{~mm} @ 85 \mathrm{~mm} \mathrm{c} / \mathrm{c}, \mathrm{f}_{\mathrm{yh}}=440$ $\mathrm{MPa}$, bottom and upper concrete cover $=25 \mathrm{~mm}$ and side cover $=$ $15 \mathrm{~mm}$.

\section{Solution:}

1. Determine if this beam satisfies the definition of a deep beam:

$\left(\frac{l_{c}}{h}\right)=2<4$ or $\left(\frac{a}{h}\right)=1<2$

Therefore, the beam is "deep beam", [ACI 318M-14(9), section 9.9.1.1] ${ }^{[1]}$

2. Draw STM of continuous deep beams CD.1F, shown Fig.4:

$h=j d+\frac{w t_{t}}{2}+\frac{w t_{b}}{2}$

$\mathrm{jd}=\mathrm{a} \tan \vartheta$

$\vartheta=\tan ^{-1}\left(\frac{j d}{a}\right)$
$\mathrm{wt}_{\mathrm{b}}=\left(\mathrm{Cc}+\emptyset\right.$ st. $+\emptyset$ main $\left.+\frac{\text { spacing }}{2}\right) * 2=(25+6+16+$ 12.5) $* 2=119 \mathrm{~mm}$

or

$\mathrm{wt}_{\mathrm{b}}=(\mathrm{h}-\mathrm{d}) * 2=(500-440.5) * 2=119 \mathrm{~mm}$

$w t_{b}=w_{t}=119 \mathrm{~mm}$ becouse $\left(A_{s b}=A_{s t}\right)$

$\mathrm{h}=\mathrm{jd}+\frac{\mathrm{wt} \mathrm{t}_{\mathrm{t}}}{2}+\frac{\mathrm{wt} \mathrm{t}_{\mathrm{b}}}{2}$

$\mathrm{jd}=\mathrm{h}-\frac{\mathrm{wt}_{\mathrm{t}}}{2}-\frac{\mathrm{wt}_{\mathrm{b}}}{2}=500-119=381 \mathrm{~mm}$

$\vartheta=\tan ^{-1}\left(\frac{381}{500}\right)=37.31^{\circ}$

$\mathrm{w}_{\mathrm{se}}=\mathrm{wt}_{\mathrm{b}} \cos \vartheta+$ lse $\sin \vartheta$

$\mathrm{w}_{\mathrm{se}}=119 \cos 37.31+100 \sin 37.31=155.3 \mathrm{~mm}$

$\mathrm{w}_{\mathrm{si}}=\mathrm{wt}_{\mathrm{b}} \cos \vartheta+0.5 \mathrm{lsi} \sin \vartheta$

$\mathrm{w}_{\mathrm{Si}}=119 \cos 37.31+0.5 * 100 \sin 37.31=124.96 \mathrm{~mm}$

$\mathrm{w}_{\mathrm{sp}}=\mathrm{wt}_{\mathrm{t}} \cos \vartheta+0.5 \mathrm{lp} \sin \vartheta$

$\mathrm{w}_{\mathrm{sp}}=119 \cos 37.31+0.5 * 100 \sin 37.31=124.96 \mathrm{~mm}$

$\mathrm{w}_{\mathrm{es}}=\frac{\mathrm{w}_{\mathrm{se}}}{2}+\frac{\mathrm{w}_{\mathrm{sp}}}{2}=\mathrm{wt}_{\mathrm{b}} \cos \vartheta+\frac{\mathrm{lse}+0.5 \mathrm{lp}}{2} \sin \vartheta$

$\mathrm{w}_{\mathrm{es}}=119 \cos 37.31+\frac{100+0.5 * 100}{2} \sin 37.31=140.13 \mathrm{~mm}$

$\mathrm{w}_{\mathrm{is}}=\frac{\mathrm{w}_{\mathrm{si}}}{2}+\frac{\mathrm{w}_{\mathrm{sp}}}{2}=\mathrm{wt}_{\mathrm{b}} \cos \vartheta+\frac{\mathrm{lsi}+\mathrm{lp}}{4} \sin \vartheta$

$\mathrm{W}_{\mathrm{is}}=119 \cos 37.31+\frac{100+100}{4} \sin 37.31=124.96 \mathrm{~mm}$

$\mathrm{F}_{\text {nes }}=0.85 \beta \mathrm{s} . \mathrm{f}_{\mathrm{c}^{\prime}} . \mathrm{b} \cdot \mathrm{w}_{\mathrm{es}}$, Fig. 5 -a

$\mathrm{F}_{\text {nis }}=0.85 \beta \mathrm{s}_{\mathrm{f}} \mathrm{f}_{\mathrm{c}^{\prime}}$. b. $\mathrm{w}_{\text {is }} \quad$, Fig. $5-\mathrm{b}$

$\beta s=0.75$ when $Q \geq 0.003$

$\beta s=0.6 \lambda$ when $Q<0.003$

$$
\mathrm{Q}=\frac{\mathrm{A}_{\mathrm{svi}}}{\mathrm{b}_{\mathrm{i}} * \mathrm{~s}_{\mathrm{i}}} \sin \vartheta_{\mathrm{i}}=\frac{2 * \frac{\pi}{4} * 6^{2}}{150 * 85} \sin 37.31+
$$

$\frac{2 * \frac{\pi}{4} * 6^{2}}{150 * 85} \sin (90-37.31)=0.0062$

$Q>0.003 \rightarrow \beta s=0.75$ [ACI 318M-14, Table 23.4.3] [1]

$\mathrm{F}_{\text {nes }}=0.85 * 0.75 * 30 * 150 * 140.13=402 \mathrm{kN}$

$\mathrm{F}_{\text {nes }} \cos \vartheta=319.74 \mathrm{kN}$

$\mathrm{F}_{\text {nis }}=0.85 * 0.75 * 30 * 150 * 124.96=358.5 \mathrm{kN}$

$\mathrm{F}_{\text {nis }} \cos \vartheta=285.14 \mathrm{kN}$

Check Capacity

For node $A$ (CCT) inclind face , Fig.5-c

$\mathrm{F}_{\mathrm{nA}}=0.85 \beta \mathrm{n} . \mathrm{f}_{\mathrm{c}^{\prime}} . \mathrm{A}_{\mathrm{nA}}$

$\beta \mathrm{n}=0.8$ for CCT [ACI 318M-14, Table 23.9.2] [1]

$\mathrm{A}_{\mathrm{nA}}=\min \left[\mathrm{w}_{\mathrm{se}} * \mathrm{~b},\left(\sqrt{\mathrm{wt}_{\mathrm{b}}{ }^{2}+\mathrm{lse}^{2}}\right) * \mathrm{~b}\right]$

$A_{n A}=\min \left[155.3 * 150,\left(\sqrt{119^{2}+100^{2}}\right) * 150\right]$

$A_{n A}=\min [23295,23315.7] \mathrm{mm}^{2}$

$\mathrm{F}_{\mathrm{nA}}=0.85 * 0.8 * 30 * 23295=475.22 \mathrm{kN}$

$\mathrm{F}_{\mathrm{nA}}>\mathrm{F}_{\text {nes }} \quad$ O.K. 


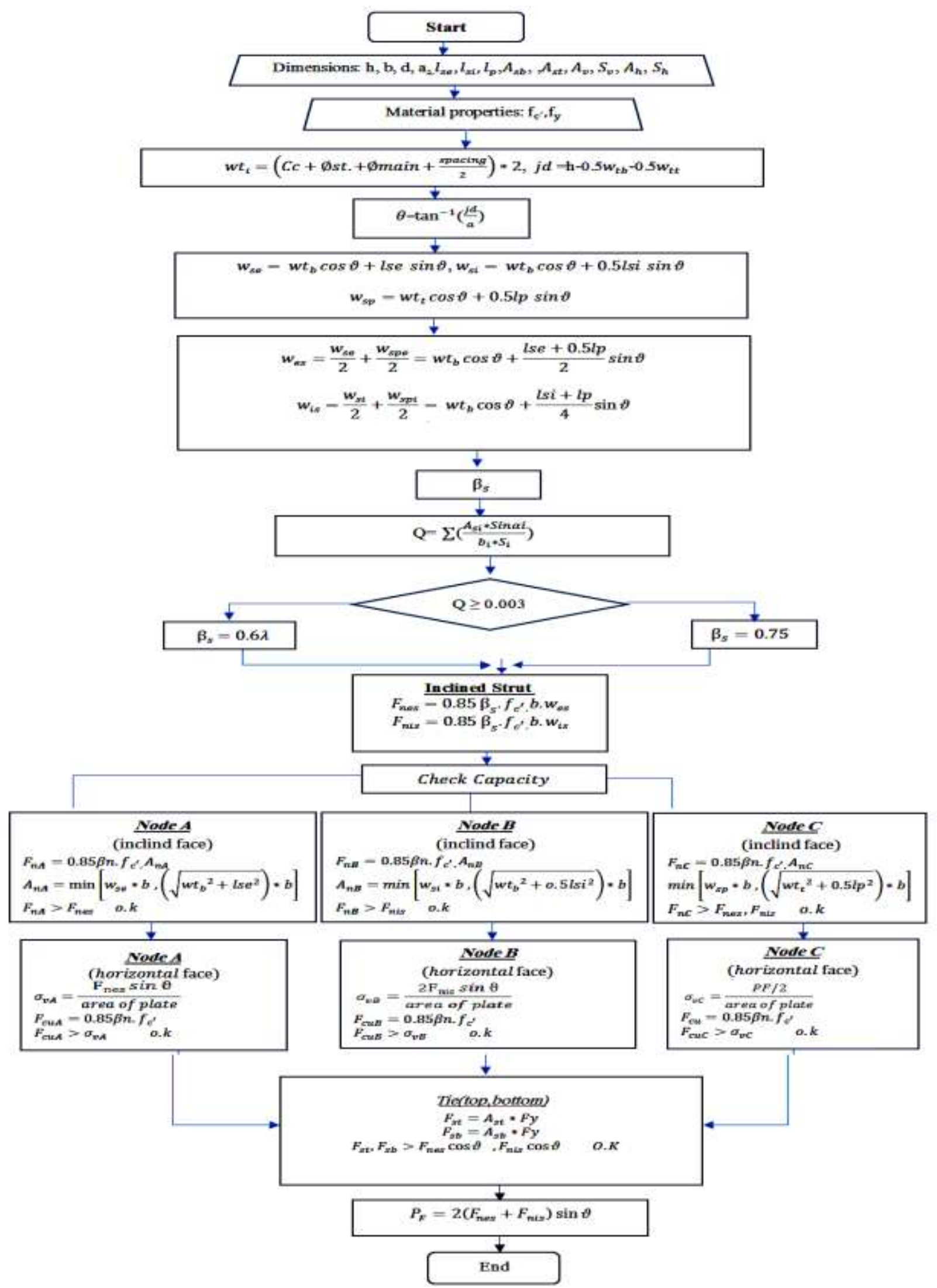

Fig. 2: STM Flow chart for light weight and normal weight reinforced concrete continuous deep beams subjected to 1-concentrated force

\section{Horizotal face}

$\sigma_{\mathrm{vA}}=\frac{\mathrm{F}_{\text {nes }} \sin \vartheta}{\text { area of plate }}=\frac{243.7 * 1000}{150 * 100}=16.2 \mathrm{MPa}$

$\mathrm{F}_{\text {cuA }}=0.85 \beta$ n. $\mathrm{f}_{\mathrm{c}^{\prime}}$

$\beta \mathrm{n}=0.8$ for CCT [ACI 318M-14, Table 23.9.2] [1]

$\mathrm{F}_{\mathrm{cuA}}=0.85 * 0.8 * 30=20.4 \mathrm{MPa}$

$\mathrm{F}_{\mathrm{cuA}}>\sigma_{\mathrm{vA}}$, The dimension of plate it is $\mathrm{O} . \mathrm{K}$.
Tie 1

$\mathrm{F}_{\mathrm{sb}}=\mathrm{A}_{\mathrm{sb}} * \mathrm{Fy}=4 * \frac{\pi}{4} 16^{2} * 500=402.12 \mathrm{kN}>$

$\mathrm{F}_{\text {nes }} \cos \vartheta \quad$ O. K.

For node $B$ [CCT] inclind face,Fig. 5-d

$\mathrm{F}_{\mathrm{nB}}=0.85 \beta \mathrm{n} . \mathrm{f}_{\mathrm{c}^{\prime}} . \mathrm{A}_{\mathrm{nB}}$

$\beta n=0.8$ for CCT [ACI 318M-14, Table 23.9.2] [1] 
$\mathrm{A}_{\mathrm{nB}}=\min \left[\mathrm{w}_{\mathrm{si}} * \mathrm{~b},\left(\sqrt{\mathrm{wt}_{\mathrm{b}}^{2}+0.5 \mathrm{lsi}^{2}}\right) * \mathrm{~b}\right]$

$A_{n B}=\min \left[124.96 * 150,\left(\sqrt{119^{2}+50^{2}}\right) * 150\right]$

$\mathrm{A}_{\mathrm{nB}}=\min [18744,19361.62] \mathrm{mm}^{2}$

$\mathrm{F}_{\mathrm{nB}}=0.85 * 0.8 * 30 * 18744=382.4 \mathrm{kN}$

$\mathrm{F}_{\mathrm{nB}}>\mathrm{F}_{\text {nis }} \quad \mathrm{O} . \mathrm{K}$.

Horizotal face

$\sigma_{\mathrm{vB}}=\frac{2 \mathrm{~F}_{\text {nis }} \sin \vartheta}{\text { area of plate }}=\frac{434.6 * 1000}{150 * 100}=29 \mathrm{MPa}$

$\mathrm{F}_{\mathrm{cuB}}=0.85 \beta \mathrm{n} . \mathrm{f}_{\mathrm{c}^{\prime}}$

$\beta \mathrm{n}=0.8$ for CCT [ACI 318M-14, Table 23.9.2] [1]

$\mathrm{F}_{\text {cuB }}=0.85 * 0.8 * 30=20.4 \mathrm{MPa}$

$\sigma_{\mathrm{vB}}>\mathrm{F}_{\mathrm{cuB}}\left(\begin{array}{c}\text { increase dimension of plate or using nodal } \\ \text { reinforcement to prevent premature failure }\end{array}\right)$

For node $\mathrm{C}$ [CCC/CCT] inclind face ,Fig. 5-e

$\mathrm{F}_{\mathrm{nC}}=0.85 \beta \mathrm{n} . \mathrm{f}_{\mathrm{c}^{\prime}} \mathrm{A}_{\mathrm{nC}}$

$(\beta \mathrm{n}=1)$ for CCC [ACI 318M-14, Table 23.9.2] [1]

$(\beta \mathrm{n}=0.8)$ for CCT [ACI 318M-14, Table 23.9.2] [1]

$\mathrm{A}_{\mathrm{nC}}=\min \left[\mathrm{w}_{\mathrm{sp}} * \mathrm{~b},\left(\sqrt{\mathrm{wt}_{\mathrm{t}}^{2}+0.5 \mathrm{lp}^{2}}\right) * \mathrm{~b}\right]$

$A_{\mathrm{nC}}=\min \left[124.96 * 150,\left(\sqrt{119^{2}+50^{2}}\right) * 150\right]$

$\mathrm{A}_{\mathrm{nC}}=\min [18744,19361.62] \mathrm{mm}^{2}$

$\mathrm{F}_{\text {nce }}=0.85 * 1 * 30 * 18744=477.98 \mathrm{kN}>\mathrm{F}_{\mathrm{nes}}$

$\mathrm{F}_{\text {nci }}=0.85 * 0.8 * 30 * 18744=382.4 \mathrm{kN}>\mathrm{F}_{\text {nis }}$

O. K.

Horizotal face

$\sigma_{\mathrm{vC}}=\frac{\mathrm{PF} / 2}{\text { area of plate }}=\frac{446.1 * 1000}{150 * 100}=29.74 \mathrm{MPa}$

$\mathrm{F}_{\mathrm{cuC}}=0.85 \beta \mathrm{n} . \mathrm{f}_{\mathrm{c}^{\prime}}$

$\beta \mathrm{n}=0.8$ for CCT [ACI 318M-14, Table 23.9.2] [1]

$\mathrm{F}_{\text {cuC }}=0.85 * 0.8 * 30=20.4 \mathrm{MPa}$

$\sigma_{\mathrm{vC}}>\mathrm{F}_{\mathrm{cuC}}\left(\begin{array}{l}\text { increase dimension of plate or using nodal } \\ \text { reinforcement to prevent premature failure }\end{array}\right)$

Tie 2

$\mathrm{F}_{\mathrm{st}}=\mathrm{A}_{\mathrm{st}} * \mathrm{Fy}=4 * \frac{\pi}{4} 16^{2} * 500=402.12 \mathrm{kN}>$

$\begin{array}{ll}\mathrm{F}_{\text {nis }} \cos \vartheta & \text { O. K. }\end{array}$

$$
P_{F}=2\left(F_{n e s}+F_{n i s}\right) \sin \vartheta=2(402+358.5) \sin 37.31
$$$$
=921.92 \mathrm{kN}
$$

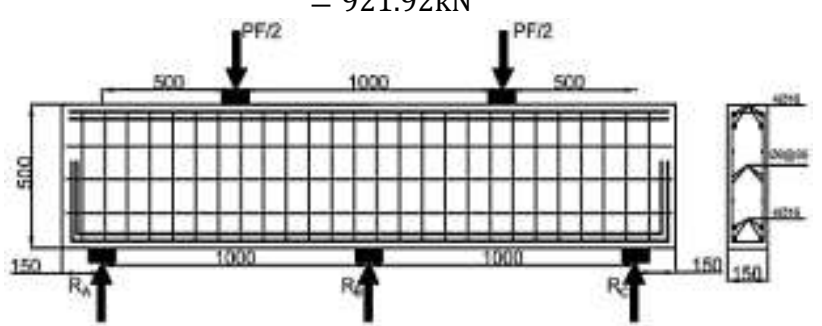

Fig. 3: Details of CD.1F, all dimensions in mm

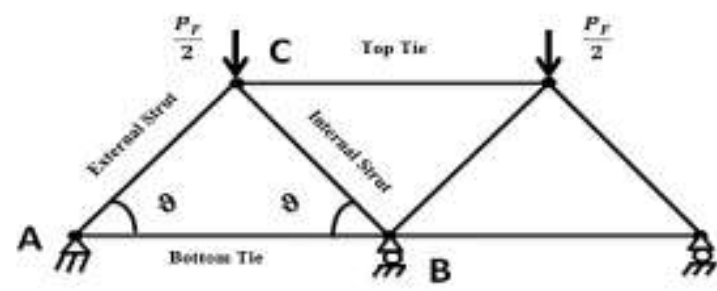

Fig. 4: Strut and Tie model for CD.1F

\subsection{Two Conce9trated Forces}

Fig.6 shows the principal stress paths and the assumed truss under 2-concentrated forces in continuous deep beam specimen CD.2F. According to the shear provisions of the ACI 318M-14 design code, same as in the case of 1-concentrated force, the geometry should be conformed to the deep beam definition $l_{n} \leq 4 h$ [1]. Similarly, the minimum web reinforcement ratios for both horizontal and vertical ones should be 0.0025 with the maximum spacing of $d / 5$, which is not more than $300 \mathrm{~mm}$ [1]. Finally, capacity was checked for each node face, the idealized bottle shape diagonal strut, in addition to top and bottom ties.
To analyze the deep beam with two concentrated forces, the steps shown in Fig.7 may be followed. Details for the specimen CD.2F are shown in Fig. 8.
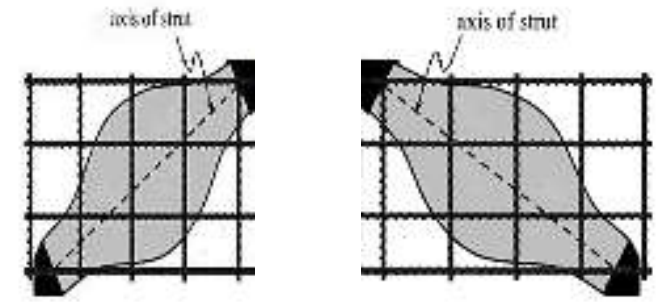

Fig. 5-a: External strut

Fig.5-b: Internal strut

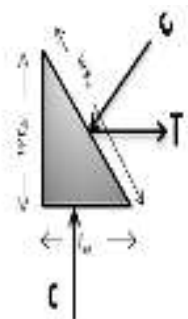

Fig. 5-c: Faces of support nodal

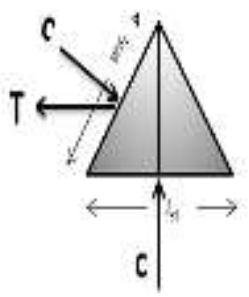

Fig. 5-d: Faces of support nodal

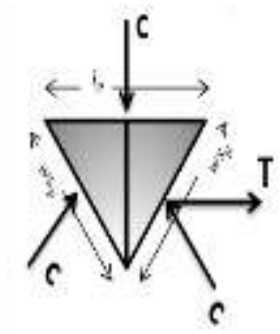

Fig.5-e: Faces of load nodal zone Fig. 5: Nodal zones and struts for CD.1F.

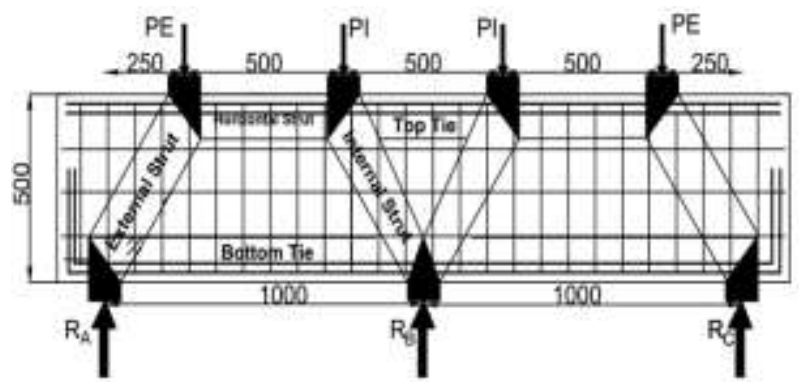

Fig. 6: The principal stress paths and the assumed truss for CD.2F, all dimensions in $\mathrm{mm}$.

\subsubsection{Numerical Example No.2, Two Concentrated Forces:}

Sample calculations for predicting failure load depending on strut and tie method for reinforced concrete deep beam that subjected to two central concentrated forces is presented here.

$\mathrm{h}=500 \mathrm{~mm}, l c=1000 \mathrm{~mm}, \mathrm{~d}=44.5 \mathrm{~mm}, a=250 \mathrm{~mm}, b_{w}=150 \mathrm{~mm}, \mathrm{f}^{\prime} \mathrm{c}$ $=30 \mathrm{MPa}$, bearing plate dimensions $=(100 * 150) \mathrm{mm}$, main longitudinal top and bottom reinforcement $=4 \phi 16 \mathrm{~mm}, \mathrm{f}_{\mathrm{y}}=500$ $\mathrm{MPa}$, vertical web reinforcement $=\phi 6 \mathrm{~mm} @ 85 \mathrm{~mm} \mathrm{c} / \mathrm{c}, \mathrm{f}_{\mathrm{yv}}=440$ $\mathrm{MPa}$, horizontal web reinforcement $=\phi 6 \mathrm{~mm} @ 85 \mathrm{~mm} \mathrm{c} / \mathrm{c}, \mathrm{f}_{\mathrm{yh}}=440$ $\mathrm{MPa}$, bottom and upper covers $=25 \mathrm{~mm}$ in addition to side cover of $15 \mathrm{~mm}$. 


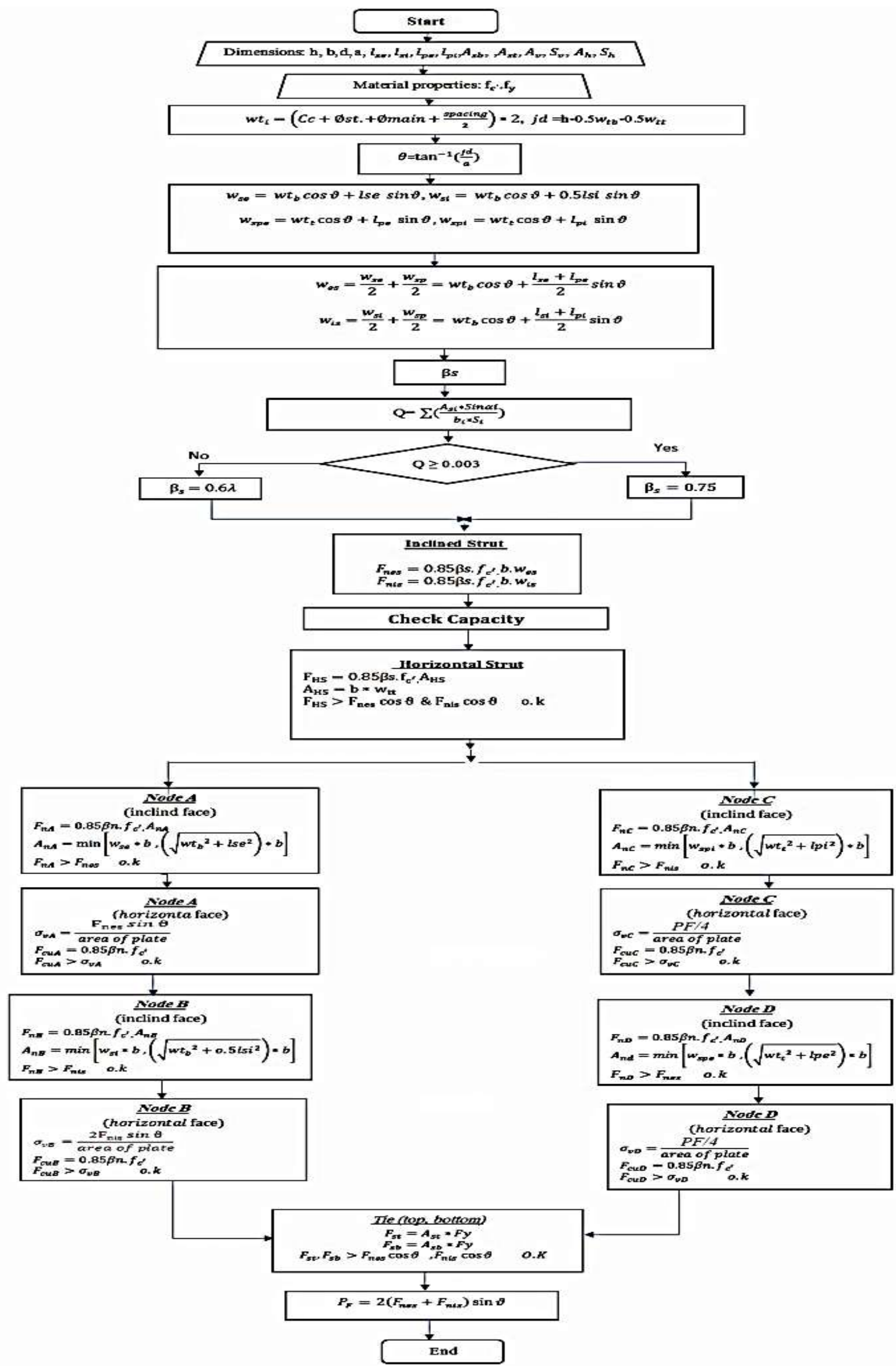

Fig. 7: STM Flow chart for light weight and normal weight reinforced concrete continuous deep beams subjected to 2-concentrated forces 


\section{Solution:}

satisfies the definition of a deep beam:

$\left(\frac{l_{c}}{h}\right)=2<4 \quad$ or $\quad\left(\frac{a}{h}\right)=0.5<2$

Therefore, the beam is "deep beam", [ACI 318M-14(9), section 9.9.1.1] [1]

2. Draw STM of deep beam CD.2F ,Fig. 9:

$\mathrm{h}=\mathrm{jd}+\frac{w \mathrm{t}_{\mathrm{t}}}{2}+\frac{w \mathrm{t}_{\mathrm{b}}}{2}$

$\mathrm{jd}=\mathrm{a} \tan \vartheta$

$\vartheta=\tan ^{-1}\left(\frac{\mathrm{jd}}{\mathrm{a}}\right)$

$\mathrm{wt}_{\mathrm{b}}=\left(\mathrm{Cc}+\emptyset\right.$ st. $+\emptyset$ main $\left.+\frac{\text { spacing }}{2}\right) * 2=(25+6+16+$

$12.5) * 2=119 \mathrm{~mm}$

$\mathrm{wt}_{\mathrm{b}}=(\mathrm{h}-\mathrm{d}) * 2=(500-440.5) * 2=119 \mathrm{~mm}$

$\mathrm{wt}_{\mathrm{b}}=\mathrm{wt}_{\mathrm{t}}=119 \mathrm{~mm}$ becouse $\left(\mathrm{A}_{\mathrm{sb}}=\mathrm{A}_{\mathrm{st}}\right)$

$\mathrm{h}=\mathrm{jd}+\frac{\mathrm{wt}}{2}+\frac{w t_{\mathrm{b}}}{2}$

$\mathrm{jd}=\mathrm{h}-\frac{\mathrm{wt}_{\mathrm{t}}}{2}-\frac{\mathrm{wt}_{\mathrm{b}}}{2}=500-119=381 \mathrm{~mm}$

$\vartheta=\tan ^{-1}\left(\frac{381}{250}\right)=56.73^{\circ}$

$\mathrm{w}_{\mathrm{se}}=\mathrm{wt}_{\mathrm{b}} \cos \vartheta+$ lse $\sin \vartheta$

$\mathrm{w}_{\mathrm{se}}=119 \cos 56.73+100 \sin 56.73=148.9 \mathrm{~mm}$

$\mathrm{w}_{\mathrm{si}}=\mathrm{wt}_{\mathrm{b}} \cos \vartheta+0.5 \mathrm{lsi} \sin \vartheta$

$\mathrm{w}_{\mathrm{si}}=119 \cos 56.73+0.5 * 100 \sin 56.73=107.09 \mathrm{~mm}$

$\mathrm{w}_{\text {spe }}=\mathrm{wt}_{\mathrm{t}} \cos \vartheta+\mathrm{l}_{\mathrm{pe}} \sin \vartheta$

$\mathrm{w}_{\text {spe }}=119 \cos 56.73+100 \sin 56.73=148.9 \mathrm{~mm}$

$\mathrm{w}_{\mathrm{spi}}=\mathrm{wt}_{\mathrm{t}} \cos \vartheta+\mathrm{l}_{\mathrm{pi}} \sin \vartheta$

$\mathrm{w}_{\mathrm{spi}}=119 \cos 56.73+100 \sin 56.73=148.9 \mathrm{~mm}$

$\mathrm{w}_{\mathrm{es}}=\frac{\mathrm{w}_{\mathrm{se}}}{2}+\frac{\mathrm{w}_{\text {spe }}}{2}=\mathrm{wt}_{\mathrm{b}} \cos \vartheta+\frac{\text { lse }+ \text { lpe }}{2} \sin \vartheta$

$\mathrm{w}_{\mathrm{es}}=119 \cos 56.73+\frac{100+100}{2} \sin 56.73=148.9 \mathrm{~mm}$

$\mathrm{w}_{\mathrm{is}}=\frac{\mathrm{w}_{\mathrm{si}}}{2}+\frac{\mathrm{w}_{\mathrm{spi}}}{2}=\mathrm{wt}_{\mathrm{b}} \cos \vartheta+\frac{0.51 \mathrm{si}+\mathrm{lpi}}{2} \sin \vartheta$

$\mathrm{w}_{\mathrm{is}}=119 \cos 56.73+\frac{50+100}{2} \sin 56.73=128 \mathrm{~mm}$

$\mathrm{F}_{\text {nes }}=0.85 \beta \mathrm{s} . \mathrm{f}_{\mathrm{c}^{\prime}} . \mathrm{b} \cdot \mathrm{w}_{\mathrm{es}}$

$\mathrm{F}_{\text {nis }}=0.85 \beta \mathrm{s} . \mathrm{f}_{\mathrm{c}^{\prime}}$. b. $\mathrm{w}_{\text {is }}$

$\beta s=0.75$ when $\mathrm{Q} \geq 0.003$

$\beta s=0.6 \lambda$ when $Q<0.003$

$\mathrm{Q}=\frac{\mathrm{A}_{\mathrm{svi}}}{\mathrm{b}_{\mathrm{i}} * \mathrm{~s}_{\mathrm{i}}} \sin \vartheta_{\mathrm{i}}=\frac{2 * \frac{\pi}{4} * 6^{2}}{150 * 85} \sin (56.73)+\frac{2 * \frac{\pi}{4} * 6^{2}}{150 * 85} \sin (90-56.73)=$ 0.0061

$\mathrm{Q}>0.003 \rightarrow \beta \mathrm{s}=0.75$ [ACI 318M-14, Table 23.4.3] [1]

$\mathrm{F}_{\text {nes }}=0.85 * 0.75 * 30 * 150 * 148.9=427.16 \mathrm{kN}$

$\mathrm{F}_{\text {nes }} \cos \vartheta=234.33 \mathrm{kN}$

$\mathrm{F}_{\text {nis }}=0.85 * 0.75 * 30 * 150 * 128=367.2 \mathrm{kN}$

$\mathrm{F}_{\text {nis }} \cos \vartheta=201.44 \mathrm{kN}$

Check Capacity

For Horizontal Strut ,Fig. 10-a

$\mathrm{F}_{\mathrm{HS}}=0.85 \beta \mathrm{s} . \mathrm{f}_{\mathrm{c}^{\prime}} \mathrm{A}_{\mathrm{HS}}$

$\beta s=1$ [ACI 318M-14, Table 23.4.3] [1]

$\mathrm{A}_{\mathrm{HS}}=119 * 150=17850 \mathrm{~mm}^{2}$

$\mathrm{F}_{\mathrm{HS}}=0.85 * 1 * 30 * 17850=455.2 \mathrm{kN}$

$F_{H S}+$ Tie $2>F_{n i s} \cos \vartheta \quad 0 \mathrm{~K}$

For node $A$ (CCT) inclind face, Fig. 10-b

$\mathrm{F}_{\mathrm{nA}}=0.85 \beta \mathrm{n} . \mathrm{f}_{\mathrm{c}^{\prime}} . \mathrm{A}_{\mathrm{nA}}$

$\beta \mathrm{n}=0.8$ for CCT [ACI 318M-14, Table 23.9.2] [1]

$\mathrm{A}_{\mathrm{nA}}=\min \left[\mathrm{w}_{\mathrm{se}} * \mathrm{~b},\left(\sqrt{\mathrm{wt}_{\mathrm{b}}^{2}+\mathrm{lse}^{2}}\right) * \mathrm{~b}\right]$

$\mathrm{A}_{\mathrm{nA}}=\min \left[148.9 * 150,\left(\sqrt{119^{2}+100^{2}}\right) * 150\right]$

$\mathrm{A}_{\mathrm{nA}}=\min [22335,23315.7] \mathrm{mm}^{2}$

$F_{n A}=0.85 * 0.8 * 30 * 22335=455.63 \mathrm{kN}$

$F_{n A}>F_{n e s} \quad$ o. $k$

Horizotal face

$\sigma_{\mathrm{vA}}=\frac{\mathrm{F}_{\text {nes }} \sin \vartheta}{\text { area of plate }}=\frac{357.15 * 1000}{150 * 100}=23.8 \mathrm{MPa}$

$\mathrm{F}_{\text {cuA }}=0.85 \beta \mathrm{n} \cdot \mathrm{f}_{\mathrm{c}^{\prime}}$

$\beta \mathrm{n}=0.8$ for CCT [ACI 318M-14, Table 23.9.2] [1]

$\mathrm{F}_{\mathrm{cuA}}=0.85 * 0.8 * 30=20.4 \mathrm{MPa}$

$\mathrm{F}_{\mathrm{cuA}}<\sigma_{\mathrm{vA}}\left(\begin{array}{c}\text { increasing dimensions of plate or using nodal } \\ \text { reinforcement to prevent premature failure }\end{array}\right)$

Tie 1
1. Determine if this beam

$\mathrm{F}_{\mathrm{sb}}=\mathrm{A}_{\mathrm{sb}} * \mathrm{Fy}=4 * \frac{\pi}{4} 16^{2} * 500=402.12 \mathrm{kN}>$

$\mathrm{F}_{\text {nes }} \cos \vartheta \quad \mathrm{OK}$

For node $B$ [CCT] inclind face, Fig.10-c.

$\mathrm{F}_{\mathrm{nB}}=0.85 \beta \mathrm{n} . \mathrm{f}_{\mathrm{c}^{\prime}} . \mathrm{A}_{\mathrm{nB}}$

$\beta \mathrm{n}=0.8$ for CCT [ACI 318M-14, Table 23.9.2] [1]

$A_{n B}=\min \left[w_{s i} * b,\left(\sqrt{w_{b_{b}}^{2}+0.51 s^{2}}\right) * b\right]$

$A_{n B}=\min \left[107.09 * 150,\left(\sqrt{119^{2}+50^{2}}\right) * 150\right]$

$A_{\mathrm{nB}}=\min [16063.5,19361.62] \mathrm{mm}^{2}$

$\mathrm{F}_{\mathrm{nB}}=0.85 * 0.8 * 30 * 16063.5=327.7 \mathrm{kN}$

$\mathrm{F}_{\mathrm{nB}}<\mathrm{F}_{\text {nis }}\left(\begin{array}{c}\text { increase dimension of plate or using nodal } \\ \text { reinforcement to strenghning nodal zone }\end{array}\right)$

Horizotal face

$\sigma_{\mathrm{vB}}=\frac{2 \mathrm{~F}_{\text {nis }} \sin \vartheta}{\text { area of plate }}=\frac{614.03 * 1000}{150 * 100}=40.9 \mathrm{MPa}$

$\mathrm{F}_{\text {cuB }}=0.85 \beta \mathrm{n} . \mathrm{f}_{\mathrm{c}^{\prime}}$

$\beta \mathrm{n}=0.8$ for CCT [ACI 318M-14, Table 23.9.2] [1]

$\mathrm{F}_{\text {cuB }}=0.85 * 0.8 * 30=20.4 \mathrm{MPa}$

$\sigma_{\mathrm{vB}}>\mathrm{F}_{\mathrm{cuB}}\left(\begin{array}{c}\text { increasing dimensions of plate or using nodal } \\ \text { reinforcement to prevent premature failure }\end{array}\right)$

For node $C$ [CCT] inclind face ,Fig. 10-d.

$\mathrm{F}_{\mathrm{nC}}=0.85 \beta \mathrm{n} . \mathrm{f}_{\mathrm{c}^{\prime}} \cdot \mathrm{A}_{\mathrm{nC}}$

$\beta \mathrm{n}=0.8$ for CCT [ACI 318M-14, Table 23.9.2] [1]

$\mathrm{A}_{\mathrm{nC}}=\min \left[\mathrm{w}_{\mathrm{spi}} * \mathrm{~b},\left(\sqrt{\mathrm{wt}_{\mathrm{t}}^{2}+\mathrm{lpi}^{2}}\right) * \mathrm{~b}\right]$

$A_{\mathrm{nC}}=\min \left[148.9 * 150,\left(\sqrt{119^{2}+100^{2}}\right) * 150\right]$

$\mathrm{A}_{\mathrm{nC}}=\min [22335,23315.7] \mathrm{mm}^{2}$

$\mathrm{F}_{\mathrm{nC}}=0.85 * 0.8 * 30 * 22335=455.63 \mathrm{kN}$

$\mathrm{F}_{\mathrm{nC}}>\mathrm{F}_{\text {nis }} \quad \mathrm{O} . \mathrm{K}$.

Horizotal face

$\sigma_{\mathrm{vC}}=\frac{\mathrm{PF} / 4}{\text { area of plate }}=\frac{332.1 * 1000}{150 * 100}=22.14 \mathrm{MPa}$

$\mathrm{F}_{\text {cuC }}=0.85 \beta \mathrm{n} . \mathrm{f}_{\mathrm{c}^{\prime}}$

$\beta \mathrm{n}=0.8$ for CCT [ACI 318M-14, Table 23.9.2] [1]

$\mathrm{F}_{\text {cuC }}=0.85 * 0.8 * 30=20.4 \mathrm{MPa}$

$\mathrm{F}_{\mathrm{cuC}}<\sigma_{\mathrm{vC}} \quad\left(\begin{array}{c}\text { increasing dimensions of plate or using nodal } \\ \text { reinforcement to prevent premature failure }\end{array}\right)$

For node $D$ [CCC] inclind face ,Fig.10-e.

$\mathrm{F}_{\mathrm{nD}}=0.85 \beta \mathrm{n} . \mathrm{f}_{\mathrm{c}^{\prime}} \mathrm{A}_{\mathrm{nD}}$

$\beta \mathrm{n}=1$ for CCC [ACI 318M-14, Table 23.9.2] [1]

$\mathrm{A}_{\mathrm{nD}}=\min \left[\mathrm{w}_{\text {spe }} * \mathrm{~b},\left(\sqrt{\mathrm{wt}_{\mathrm{t}}^{2}+\mathrm{lpe}^{2}}\right) * \mathrm{~b}\right]$

$A_{\mathrm{nD}}=\min \left[148.9 * 150,\left(\sqrt{119^{2}+100^{2}}\right) * 150\right]$

$A_{n D}=\min [22335,23315.7] \mathrm{mm}^{2}$

$\mathrm{F}_{\mathrm{nD}}=0.85 * 1 * 30 * 22335=569.5 \mathrm{kN}$

$\mathrm{F}_{\mathrm{nD}}>\mathrm{F}_{\text {nes }} \quad \mathrm{O} . \mathrm{K}$.

Horizotal face

$\sigma_{\mathrm{vD}}=\frac{\mathrm{PF} / 4}{\text { area of plate }}=\frac{332.1 * 1000}{150 * 100}=22.14 \mathrm{MPa}$

$\mathrm{F}_{\mathrm{cuD}}=0.85 \beta \mathrm{n} . \mathrm{f}_{\mathrm{c}^{\prime}}$

$\beta \mathrm{n}=1$ for CCC [ACI 318M-14, Table 23.9.2] [1]

$\mathrm{F}_{\text {cuD }}=0.85 * 1 * 30=25.5 \mathrm{MPa}$

$\mathrm{F}_{\mathrm{cuD}}>\sigma_{\mathrm{vD}} \quad$ (The dimensions of plate are adequate)

Tie 2

$\mathrm{F}_{\mathrm{st}}=\mathrm{A}_{\mathrm{st}} * \mathrm{Fy}=4 * \frac{\pi}{4} 16^{2} * 500=402.12 \mathrm{kN}>$

$\mathrm{F}_{\text {nis }} \cos \vartheta \quad \mathrm{OK}$ $\mathrm{P}_{\mathrm{F}}=2\left(\mathrm{~F}_{\text {nes }}+\mathrm{F}_{\text {nis }}\right) \sin \vartheta=2(427.16+367.2) \sin 56.73$ $=1328.3 \mathrm{kN}$ 


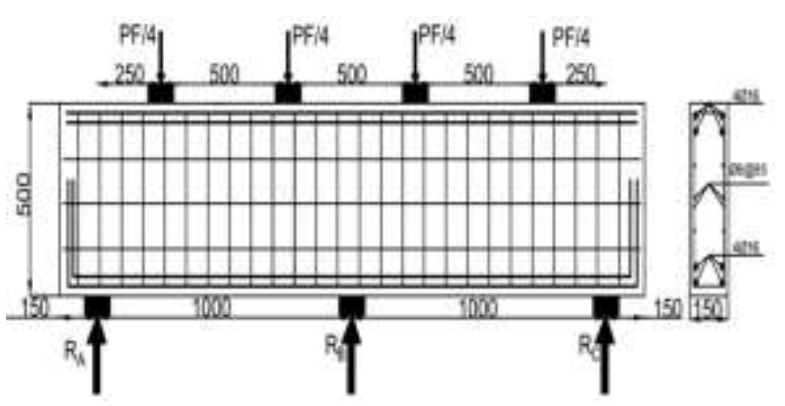

Fig. 8: Details of CD.2F, all dimensions in $\mathrm{mm}$

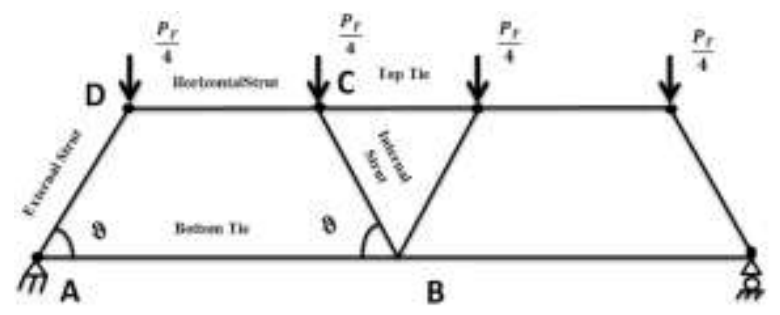

Fig. 9: Strut and Tie model for CD.2F

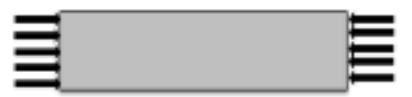

Fig. 10-a: Horizontal strut

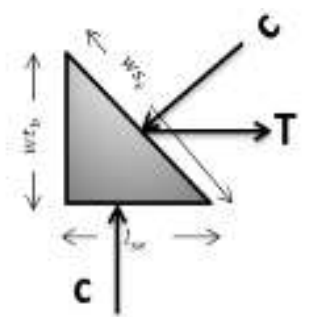

Fig. 10-b:Faces of support nodal zone

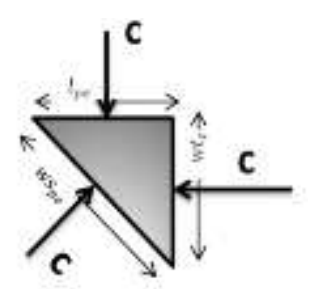

Fig.10-e: Faces of load nodal zone

Fig. 10: Nodal zones and struts for CD 2

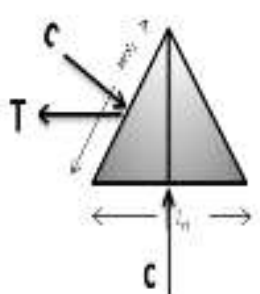

Fig. 10-c: Faces of support nodal zone

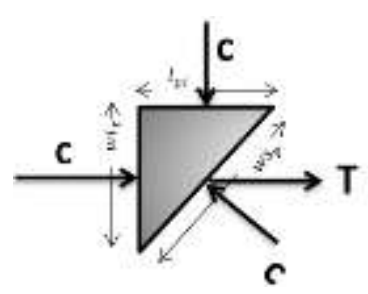

Fig.10-d: Faces of load nodal zone

\subsubsection{Uniformly Distributed Load:}

Many researchers went to the conclusion that when deep beam is subjected to uniformly distributed load, it could be considered as deep beam under two concentrated forces that should equal to the uniformly distributed load in value [14-16]. Fig. 11 shows the principal stress paths in continuous deep beam subjected to uniformly distributed load. It is worth to mention that the uniformly distributed load can be substituted by equivalent two equal forces or equivalent two unequal forces for each span. This substitution is allowed, only if the agreement of the maximum moments, the most fundamental value in the Strut-Tie model application, of the both systems is guaranteed. Fig.12-a, Fig.12-b and 1Fig.2-c. show bending moment for uniformly distributed load, two equivalent equal and unequal concentrated forces, respectively. Based on that, the prediction of strength capacity for the reinforced concrete continuous deep beam specimen subjected to uniformly distributed loading CD.UL shown in Fig.12-b and Fig.8. can be obtained by the same procedure shown in Fig.7. It was considered that the equivalent two concentrated forces are equal, so the strength capacity can be calculated by the followings:

Ultimate capacity load of continuous deep beam is $\mathrm{P}_{\mathrm{F}}=2\left(\mathrm{~F}_{\mathrm{nes}}+\mathrm{F}_{\mathrm{nis}}\right) \sin \vartheta=2(367.2+$

$307.21) \sin 56.73=1328.3 \mathrm{kN} \rightarrow \mathrm{W}_{\mathrm{F}}=\frac{\mathrm{P}_{\mathrm{F}}}{L w}=$ $1328.3 \mathrm{kN} / \mathrm{m}$

\section{Effect of loading type}

Three different types of loading are applied to reinforced concrete continuous deep beams with different $\mathrm{a} / \mathrm{h}$ ratios as shown in Table 2. It is found that the model of the struts and tie was affected by loading type. In case of 1-concentrated force, the ultimate capacity decreased by about (10-33) \% when a/h increased by about (2069) \%, Fig. 13. Whereas, in case of 2-concentrated forces, the ultimate capacity decreased by about (14-25) $\%$ when a/h increased by about (19-67) \%, see Fig. 14. Finally, in case of uniformly distributed load, the ultimate capacity decreased by about (14-25) \% when a/h increased by about (19-67) \%, see Fig.15.

Table 2 also shows how an increment in ultimate capacity by about (44-70) \% happened when the applied load was changed from 1concentrated force to 2-concentrated forces or to uniformly distributed load. The difference in capacity took place because in case of the 2-concentrated forces or the uniformly distributed load the strut-tie angle increased and the length of the external and internal struts became shorter.

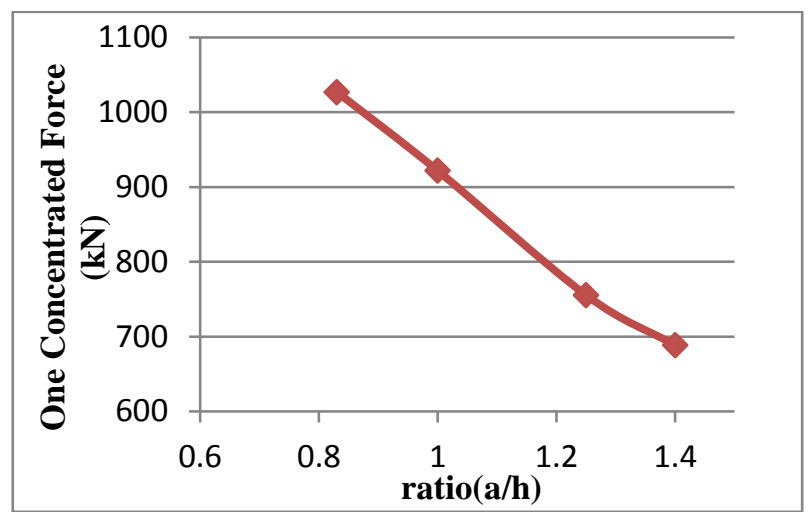

Fig. 13: Effect of $\mathrm{a} / \mathrm{h}$ ratio on the ultimate capacity for the specimens in group A

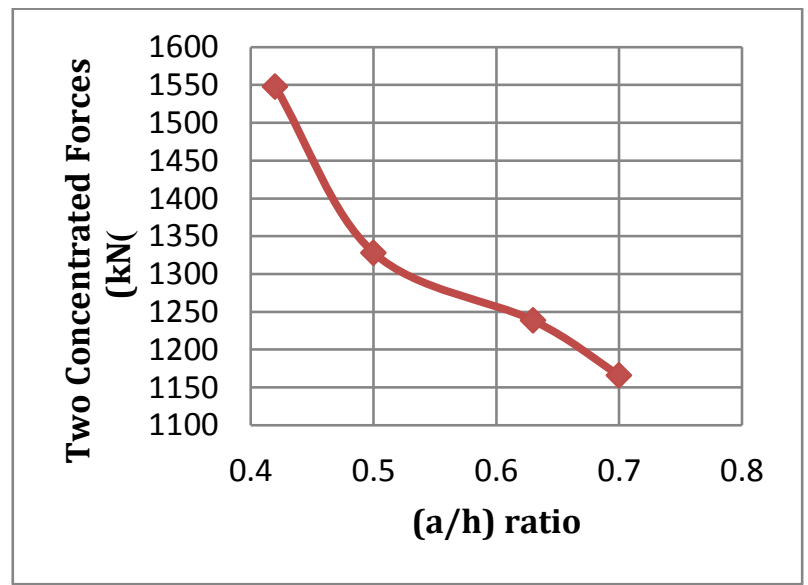

Fig. 14: Effect of a/h ratio on the ultimate capacity of the specimens in group B. 


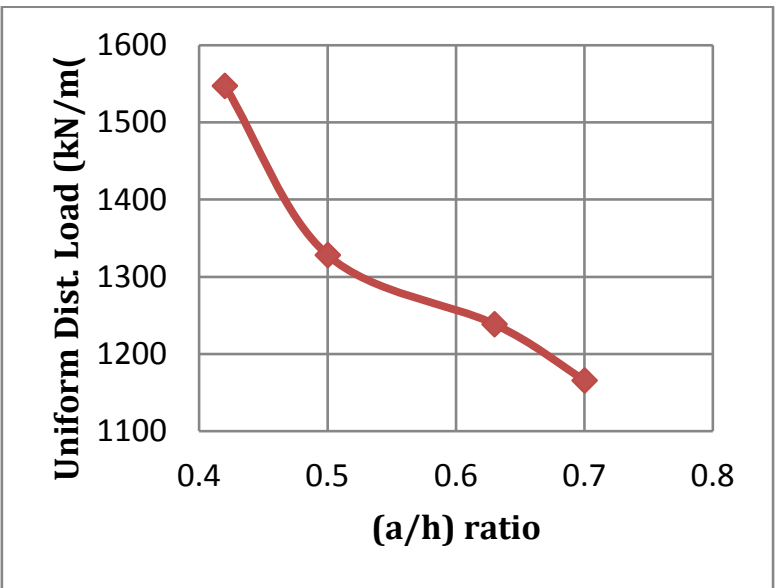

Fig.15: Effect of a/h ratio on the ultimate capacity of the specimens in group C

\section{Conclusions:}

Detailed prediction calculations for $\mathrm{RC}$ continuous deep beams that subjected to different types of loading are investigated here. It was clear that the type of loading identifies the shape of the strut and tie model, which leads to enormous differences in ultimate capacity. It was found that changing the loading type has a significant impact on the capacity for the same specimen that has the same dimensions, concrete and steel properties, in addition to the same steel amount and arrangement. In more detail, the increase in the number of concentrated forces causes an obvious increase in ultimate capacity because increasing the number of concentrated loads for the same span causes a reduction in $\mathrm{a} / \mathrm{h}$ ratio and increases the value of the strut-tie angle, which causes shortening in the length of the strut. That causes an increment in ultimate capacity by about (4470) $\%$ when the applied load was changed from 1concentrated force to 2-concentrated forces or to uniformly distributed load.

The importance of $\mathrm{a} / \mathrm{h}$ ratio urged the authors to study its effect in case of the same load type. Based on that, in case of 1-concentrated force, the ultimate load capacity decreased by about (10-33) \%, when a/h increased by about (20-69) \%, while in case of 2-concentrated forces and uniformly distributed load, the ultimate load capacity decreased by about (14-25) \% when a/h increased by about $(19-67) \%$.

Table 2: Effect of loading types on the ultimate capacity

\begin{tabular}{|c|c|c|c|c|c|c|c|c|c|}
\hline \multirow{2}{*}{ 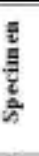 } & \multirow{2}{*}{$\frac{\hat{\sigma}}{5}$} & \multirow{2}{*}{$\begin{array}{l}\text { Load } \\
\text { Type }\end{array}$} & \multirow{2}{*}{$\begin{array}{c}\begin{array}{c}\text { Shear } \\
\text { Span } \\
\text { (mun) }\end{array} \\
a\end{array}$} & \multirow{2}{*}{$\begin{array}{c}\begin{array}{c}\text { Over:a } \\
\text { Depth } \\
\text { (man) }\end{array} \\
h\end{array}$} & \multirow{2}{*}{$\begin{array}{c}\begin{array}{c}\text { Angle } \\
\text { (degrees) }\end{array} \\
\theta\end{array}$} & \multicolumn{2}{|c|}{ Dltimate capacity } & \multirow{2}{*}{$a / b$} & \multirow{2}{*}{$\begin{array}{c}\omega_{b} \\
\text { Decrease } \\
\text { in } P_{r}\end{array}$} \\
\hline & & & & & & $\begin{array}{c}P_{F} \\
(\mathrm{kN})\end{array}$ & $\begin{array}{c}W_{F} \\
(\mathrm{kN} / \mathrm{m})\end{array}$ & & \\
\hline 1 & \multirow{4}{*}{ A } & \multirow{4}{*}{$\begin{array}{c}\text { Single } \\
\text { Concentuat } \\
\text { ed } \\
\text { Force }\end{array}$} & 500 & 600 & 39.35 & 1027,18 & - & 0.83 & - \\
\hline 2 & & & 300 & 500 & 37.31 & 921.92 & - & 1 & 10.25 \\
\hline 3 & & & 500 & 400 & 29.34 & 755.50 & $\cdot$ & 1.25 & 26.45 \\
\hline 4 & & & 500 & 360 & 25.7 & 688.62 & - & 1.4 & 33.00 \\
\hline 1 & \multirow{4}{*}{ B } & \multirow{4}{*}{$\begin{array}{c}\text { Twe } \\
\text { Concentrat } \\
\text { ed } \\
\text { forces }\end{array}$} & 250 & 600 & 60.40 & 1547.75 & - & 0.42 & - \\
\hline 2 & & & 250 & 500 & 56.73 & 1328.30 & - & 0.5 & 14.18 \\
\hline 3 & & & 250 & 400 & 48.34 & 1238.60 & - & 0.63 & 19.97 \\
\hline 4 & & & 250 & 360 & 43.95 & 1166.10 & $\cdot$ & 0.7 & 24.66 \\
\hline 1 & \multirow{4}{*}{ C } & \multirow{4}{*}{$\begin{array}{l}\text { Luiformly } \\
\text { Distributed } \\
\text { Load }\end{array}$} & 250 & 600 & 60.40 & - & 1547.75 & 0.42 & - \\
\hline 2 & & & 250 & 500 & 56.73 & - & 1328.30 & 0.5 & 14.18 \\
\hline 3 & & & 250 & 400 & 48,34 & $\cdot$ & 1238.60 & 0.63 & 19.97 \\
\hline 4 & & & 250 & 360 & 43.95 & $=$ & 1166.10 & 0.7 & 24.66 \\
\hline
\end{tabular}

\section{References}

[1] ACI Committee and American Concrete Institute. "Building code requirements for structural concrete (ACI 318-14) and commentary." American Concrete Institute, 2014

[2] Zhang, Ning, and Kang-Hai Tan. "Direct Strut-and-Tie Model for Single Span and Continuous Deep Beams." Engineering Structures 29, no. 11 (November 2007): 2987-3001. doi:10.1016/j.engstruct.2007.02.004.

[3] Rasheed, Mohammed M. "Modified Softened Strut and Tie Mode for Concrete Deep Beams." Journal of Engineering and Sustainable Development 16, no. 1 (2012): 348-361

[4] Abdul-Razzaq, Khattab Saleem. "Effect of heating on simply supported reinforced concrete deep beams." Diyala Journal of Engineering Sciences 8, no. 2 (2015): 116-133.

[5] Abdul-Razzaq, Khattab Saleem, Alaa Hussein Abed, and Hayder Ihsan Ali. "Parameters affecting load capacity of reinforced selfcompacted concrete deep beams." International Journal of Engineering 5, no. 05 (2016)

[6] Abdul-Razzaq, Khattab Saleem, Hayder I. Ali, and Mais M. AbdulKareem. "A New Strengthening Technique for Deep Beam Openings Using Steel Plates." International Journal of Applied Engineering Research 12, no. 24 (2017): 15935-15947.

[7] Abdul-Razzaq, Khattab Saleem, and Ali Mustafa Jalil. "Behavior of Reinforced Concrete Continuous Deep Beams-Literature Review." In The Second Conference of Post Graduate Researches (CPGR'2017) College of Engineering, Al-Nahrain Univ., Baghdad, Iraq-4th Oct. (2017).
[8] Noori, Abdul Qader Nihad, and Adnan Falih Ali. "Effect of Web Reinforcement on Self-Compacting Reinforced Concrete Continuous Deep Beams." Journal of Engineering and Sustainable Development 22, no. 4 (2018): 211-228.

[9] Abdul-Razzaq, Khattab Saleem, and Sarah Farhan Jebur. "Experimental Verification of Strut and Tie Method for Reinforced Concrete Deep Beams under Various Types of Loadings." Journal of Engineering and Sustainable Development 21, no. 6 (2018): 39-55.

[10] Abdul-Razzaq, Khattab Saleem, Sarah F. Jebur, and Abbas H. Mohammed. "Concrete and Steel Strengths Effect on Deep Beams with Reinforced Struts." International Journal of Applied Engineering Research 13, no. 1 (2018): 66-73

[11] Abdul-Razzaq, Khattab Saleem, and Sarah Farhan Jebur. "Suggesting Alternatives for Reinforced Concrete Deep Beams by Reinforcing Struts and Ties." MATEC Web of Conferences 120 (2017): 01004 doi:10.1051/matecconf/201712001004.

[12] Nielsen, M. P., M. W. Braestrup, B. C. Jensen, and Finn Bach. "Concrete plasticity, beam shear-shear in joints-punching shear." Special Publication (1978): 1-129

[13] B. T. Martin, David H. S., W. Wassef, T. A. Cole, and N. Bahem "Verification and implementation of strut-and-tie model in LRFD bridge design specifications" NCHRP Project, (2007).

[14] Nagarajan, Praveen, and T. M. Madhavan Pillai. "Analysis and Design of Simply Supported Deep Beams Using Strut and Tie Method." Advances in Structural Engineering 11, no. 5 (October 2008): 491-499. doi:10.1260/136943308786412050.

[15] Sam-Young, Noh, Lee Chang-Yong, and Lee Kyeong-Min. "Deep Beam Design Using Strut-Tie Model."

[16] Brown, Michael D. and Oguzhan Bayrak. "Investigation of Deep Beams with Various Load Configurations." ACI Structural Journal 104, no. 5 (2007). doi: $10.14359 / 18863$ 
